\title{
Temperature and Humidity Sensor Research Based on Monobus Technology
}

\author{
QingXiu Wu ${ }^{1, a}$, GuoFang Zhang ${ }^{1, b}$, LiLi Yan ${ }^{1, c}$ \\ ${ }^{1}$ Hainan College Of Software Technology, Qionghai, Hainan Province, China \\ awu_qingxiu@aliyun.com, bhncst@aliyun.com, chnzs02@qq.com
}

Keywords: monobus; humiture sensor; DHT11.

\begin{abstract}
A new monobus temperature and humidity sensor DHT11 has the advantages of small size, simple interface, high speed response, cost effective characteristics and so on. This paper briefly introduces the concept of Monobus, and expounds the basic principle and the control use of DHT11. The many advantages of DHT11 have high application value in automatic control and industrial and agricultural areas.
\end{abstract}

\section{Introduction}

According to the national medium and long-term science and technology development plan (2006-2020), the state will give priority to the develop precision agriculture and agricultural informatization and automation in key areas of Agriculture, the Internet of things will involve energy, finance, insurance system , and transportation, tracking, health care system and the climate system, infrastructure ,and rubber forest ecological system including agriculture and forestry. The measurement precision of traditional analog temperature and humidity sensor is not high, and requires complex calibration and calibration, as well as the complexity of the signal conditioning circuit and analog digital conversion circuit, which is very inconvenient. DHT11 is a temperature and humidity compound sensor that is calibrated digital signal output. It is the application of special acquisition technology of digital module and advanced technology of temperature and humidity sensor, and have high reliability and excellent long-term stability.

\section{Monobus technology overview}

Monobus (1-wire)technology is a kind of Fieldbus Technology developed by America Dallas semiconductor company in recent years. Unlike SPI, I2C and other standard serial data communication mode, it uses a single signal line to transmiss clock and data, its advantages of I/O resources saving, simple structure, low cost, convenient bus expansion and maintenance are more and more widely used in civilian electrical appliances, industrial control field.

Monobus is applied to system that single Master control one or more slave. When there is only single Master, system can be operated by a single node system, when there are multiple slave, system can be operated by the multi node system, Master can be a micro controller, slave can be a Monobus device. In Dallas products, this kind of single bus device have a temperature sensor, a gleam of memory, A/D converter, addressable switch etc. Compared with other such as parallel, serial and special bus, the prominent characteristics of Monobus is that address lines, data lines and control lines of the host control device synthesize into a signal line,which exchange bidirectional datas wih the machine equipment. So in the multiple control objects, the wiring of the system is simple and convenient, the overhead of smaller hardware need to compensated by relatively complex software design.

Due to the single bus system uses only a signal line as a bus, in order to ensure that each device on the bus can drive bus driver in different time, and effectively distinguish between different devices on the bus. So when making a single bus device, it preparates a only chip serial number, and identifies each device by addressing. Single bus device using CMOS technology,its power consumption is very small, if not supply power alone, only using the signal to charge a small amount of electricity when 
the bus is idle can work. The 1-wire bus is always in high potential, device hanging on top of it must be open drain or three state output, wich is not to increase the burden on the bus when it does not work. Single bus data usually communicate at a rate of $16.3 \mathrm{Kbps}$, under the speed mode, the users can set the transmission rate about $100 \mathrm{Kbps}$, generally used for control or data exchange system if they doesn't require high speed. The effect distance of single bus technology generally reached $200 \mathrm{~m}$, and allow to hang hundreds of devices..

\section{Applications of Temperature and humidity sensor}

DHT11 digital temperature and humidity sensor is a digital signal output with a calibrated temperature and humidity compound sensor. It is application specific digital module acquisition technology and the temperature and humidity sensor technology to ensure that the product has high reliability and excellent long-term stability. The sensor comprises a resistance type humidity sensitive element and a temperature measuring element is connected with NTC, and with a high performance 8 bit single chip microcomputer.Using the ZigBee module based on CC2530 the IO interrupt, IAR development environment design program to monitor the temperature and humidity sensor state.

The hardware interface. The power supply voltage of DHT11 is $35.5 \mathrm{~V}$, the sensor after power on, wait for 1 s to pass the unstable state during this period without sending any instruction. Power supply pins (VDD, GND) can be added between a 100nF capacitor, for decoupling. DATA is used for communication and synchronization between microprocessor and DHT11, using the single bus data format, a communication time is about $4 \mathrm{~ms}$, the data is divided into the decimal part from the integer part.

Design and implementation of software. Temperature and humidity is an important parameter for monitoring usually need a lot of monitoring network or in the system. Wireless monitoring network composed of a plurality of distributed wireless sensor nodes and control terminal. The wireless sensor node is responsible for temperature and humidity data acquisition and then through the wireless network to send to the network control terminal.

(1)The key source code analysis, set the CC2530 I/O state, simulation of temperature and humidity sensor sequence, wherein data line and pull resistance function open. The code below:

// Temperature and humidity definition

uchar ucharFLAG,uchartemp;

uchar shidu_shi,shidu_ge,wendu_shi,wendu_ge $=4$;

uchar ucharT_data_H,ucharT_data_L,ucharRH_data_H,ucharRH_data_L,ucharcheckdata; uchar

ucharT_data_H_temp,ucharT_data_L_temp,ucharRH_data_H_temp,ucharRH_data_L_temp,ucharc heckdata_temp;

uchar ucharcomdata;

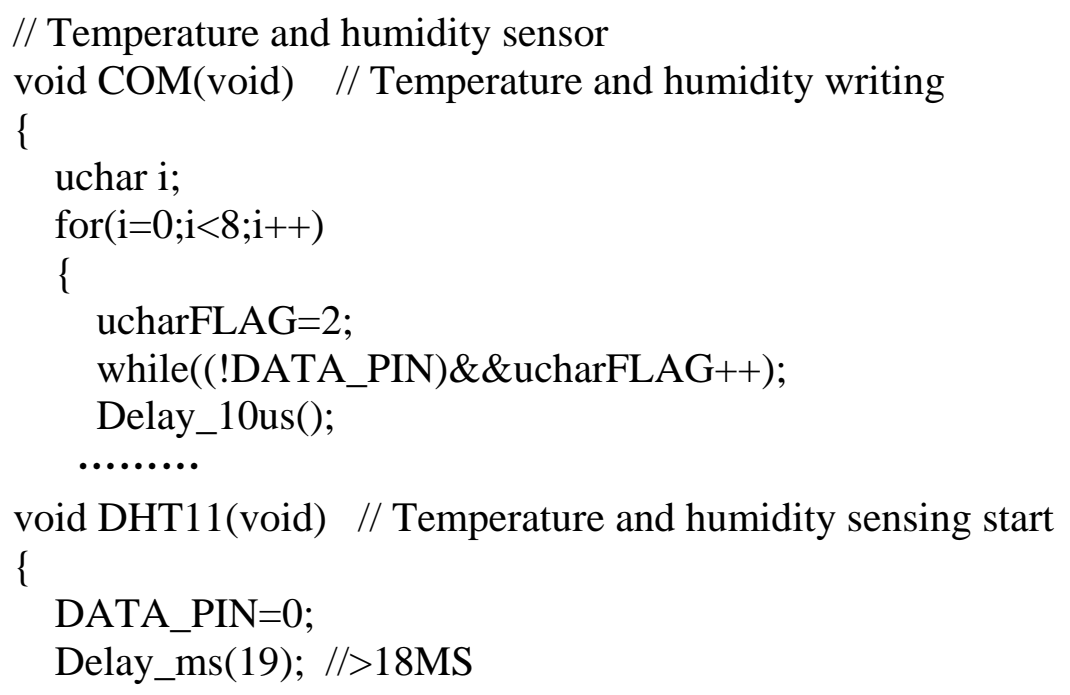


DATA_PIN=1;

P0DIR \& $=\sim 0 x 80$; / Reconfigure the IO direction of the mouth

Delay_10us();

(..........

ucharT_data_L_temp=ucharcomdata;

$\operatorname{COM}()$;

ucharcheckdata_temp=ucharcomdata;

DATA_PIN=1;

uchartemp=(ucharT_data_H_temp+ucharT_data_L_temp+ucharRH_data_H_temp+ucharRH_data_ L_temp);

if(uchartemp==ucharcheckdata_temp)

\{

ucharRH_data_H=ucharRH_data_H_temp;

\}

wendu_shi=ucharT_data_H/10;

\}

else // No successful reading, return 0

\{

wendu_shi $=0$;

wendu_ge $=0$;

shidu_shi $=0$;

\}

shidu_ge $=0$;

\}

P0DIR $\mid=0 x 80$; // IO I need to re configure

(2)The main function of the program, the code as follows:

void main(void)

\{

uchar temp[3];

uchar humidity[3];

uchar strTemp[13]="Temperature:";

uchar strHumidity[10]="Humidity:";

Delay_ms(1000); / / Let the stability of the equipment

InitUart(); // Serial port initialization

while(1)

\{

memset(temp, 0, 3);

memset(humidity, 0, 3);

DHT11(); / / The acquisition of temperature and humidity

// Will be converted into a string of temperature and humidity

temp[0]=wendu_shi+0x30;

// Temperature and humidity to get to the computer display through the serial output

UartSendString(strTemp, 12);

UartSendString(temp, 2);

UartSendString(" " ", 3);

UartSendString(strHumidity, 9); 


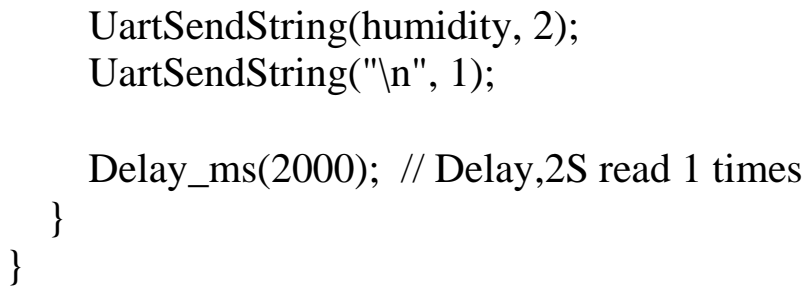

(3)The result of running the program, as shown in Fig. 1:

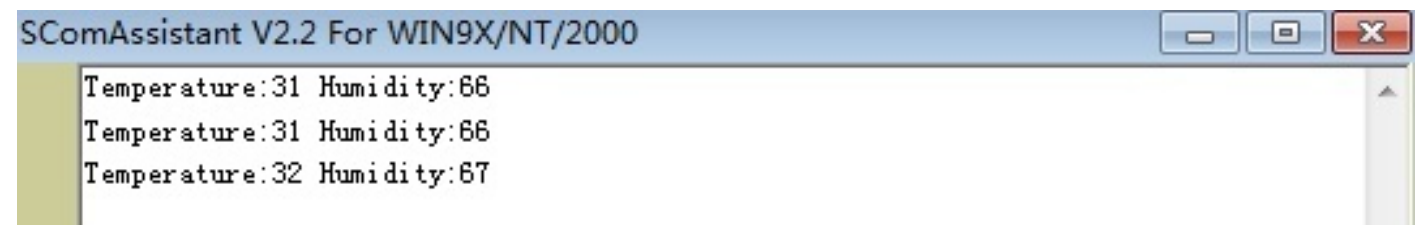

Fig. 1 program debugging results

\section{Summary}

DHT11'accuracy and stability to measure datas. Low price, small volume, accurate and stable measurement data, simple control ways of Monobus and circuit connection, which will make the DHT11 possess a good application prospect. Besides, the DHT11 has the advantages of quick response, strong anti-interference ability, high performance price ratio and so on, its application in the wireless sensor network node,and combined with wireless transceiver chip make the data acquisition node more flexible. Therefore, the design of wireless sensor network node based on DHT11 can be widely used in the laboratory, industrial, agricultural, environmental protection, health and epidemic prevention, storage and transportation, museum, greenhouse and other fields.

\section{Acknowledgement}

This work is supported by the Natural Science Foundation of Hainan Province in China (Research on rubber forest environment monitoring system based on the Internet of things No:614238) and (Application of task scheduling algorithm in cloud computing environments No:614242);Science Research Project of Colleges and Universities of Hainan Province in China (Research and implementation of network security mechanism based 6LoWPAN No: Hnky2015-79) and (Research on mobile banking security access based on SSLVPN No: Hnky2015-78).

\section{References}

[1] Chen D.H., Y.M. Liang. Design of low power consumption wireless measuring nodes for greenhouse. Process Automation Instrumentation, 2010(5):66-72.

[2] L.G. Zhou. Explain profound theories in simple language ARM7. Bei Jing: Press of BUAA, 2005.

[3] M.X. Li, K. Wang, X.Y. Gao. Application of nRF401 in environmental parameter acquisition system of greenhouse.Instrumentation, 2008(11):214-224.

[4] Y.M. Han, J.P. Zhao. Desiggn of temperature humidity wireless sensor network node based on DHT11. Journal of Jinggangshan University (Natural Science),2011,32(1):67-70.

[5] Y.M. Sun, Z.Y. Liu, et al. Low power consumption design for wireless sensor networks nodes. Microcomputer Applications, 2004 (9) :12-14..

[6] W.D. Wu, G.X. Wang, Z.X. Zhang. An design of wireless sensor network node controller of ultralower power. Journal of Shenyang Institute of Aeronautcal Engineering, 2005(6):44-46. 